\title{
NUMERICAL EVALUATION OF EFFECTIVE THERMAL PROPERTIES FOR MATERIALS WITH VARIABLE POROSITY
}

\author{
P. STAŇÁK, J. SLÁDEK, V. SLÁDEK, S. KRAHULEC
}

\begin{abstract}
In this paper a computational homogenization technique is applied to thermal analyses in porous materials. A volume fraction of pores on the microstructural level is the key factor that changes the macroscopic thermal properties. Thus, the distribution of thermal fields at the macroscopic level is analysed through the incorporation of the microstructural response on the representative volume element (RVE) assuming a uniform distribution of pores. For the numerical analysis the scaled boundary finite element method (SBFEM) is introduced to compute the thermal response of RVE. The SBFEM combines the main advantages of the finite element method (FEM) and the boundary element method (BEM). In this method, only the boundary is discretized with elements leading to the reduction of spatial dimension by one, similarly as in the BEM. It reduces computational efforts in the mesh generation and CPU time. The proposed method is used to study square RVE with a circular and elliptic pore under the thermal load. Dimensions of the pore are varied to obtain different volume fractions of matrix material. Numerical results for effective thermal conductivities obtained via SBFEM modelling show an excellent agreement with the finite element analysis using commercial software COMSOL Multiphysics.
\end{abstract}

Keywords: thermal conductivity, effective material properties, representative volume element (RVE), material porosity, scaled boundary finite element method

\section{Introduction}

Numerical modelling of heat transfer, solid mechanics, fluid flow, electromagnetics and other physical, chemical or civil engineering sciences have experienced an intense development in the past several decades. Several tools are currently available for the analysis of heat transfer problems, based on analytical formulations (Carslaw and Jaeger, 1959) or numerical methods, such as the finite difference method (FDM) (Ozisik, 1994; Juncu, 2008), the finite element method (FEM) (Bathe, 1976), the finite volume method (FVM) (Cai, Mandel and McCormick, 1991) and the boundary element method (BEM) (Brebbia, Telles and Wrobel, 1984; Ochiai, 2001; Abreu, Canelas and Mansur, 2013). In recent years, a different type of numerical method has been developed as an alternative to the well established mesh-based methods, known as meshless methods or element free methods. Among many meshless methods available the meshless MLPG method with a Heaviside step function as the test function (Atluri, 2004) has been successfully applied to solve heat conduction (Sladek et al., 2003; Abasbandy and Shirzadi, 2010) and thermoelasticity (Sladek et al., 2006; 2009). Application of the MLPG

\footnotetext{
${ }^{1}$ Institute of Construction and Architecture, Slovak Academy of Sciences, Dúbravská cesta 9, 84503 Bratislava 45, Slovak Republic, e-mail: peter.stanak@savba.sk, jan.sladek@savba.sk, vladimir.sladek@savba.sk, slavomir.krahulec@savba.sk
} 
method to analysis of a broad range of scientific problems is summarized in the review article by Sladek et al. (2013).

The above mentioned numerical methods have several drawbacks and limitations due to their inherent nature. Disadvantages of FEM are well-known, including locking phenomena in problems which involve constraints, difficulty to satisfy continuity requirements (especially in plates and shells), sensitivity to mesh distortion, etc. Many engineering problems cannot be efficiently solved by BEM because of missing fundamental solution. A drawback of meshless methods is a higher CPU time compared to regular FEM.

Undoubtedly, the scaled boundary finite element method (SBFEM) (Song and Wolf, 1997) belongs to interesting computational methods. It is a semi-analytical method developed on the assumption of separation of spatial variables. This method not only combines the main advantages of the finite element method and the boundary element method (BEM) but also has unique features of its own. The discretization of the boundary is based on the standard finite element interpolation functions. In contrast to the boundary element method, no fundamental solution is required, which permits to analyze general boundary value problems. In this method, only the boundary is discretized, which spares the human and computational efforts in a mesh generation. To model the response of structures subjected to thermal loading, Song (2006) developed a scaled boundary finite element solution where the nodal loads due to the change in temperature are treated as a non-homogeneous term in the resulting ordinary differential equations. SBFEM is very efficient in solving problems involving the fracture. The asymptotic behaviour of gradients of primary fields of any kind near the crack tip is analytically represented in the "stress" solution. Applications to thermo-piezoelectricity in the fracture mechanics have already been reported ( $\mathrm{Li}$ et al., 2015).

The permanent demand for new innovations and applications is also a driving force for the development of new materials. Recent applications of porous materials for various advanced applications also set new challenges for efficient computational modelling. The correct treatment of materials with voids seems to be an adequate tool to describe also materials with empty pores. For voided isotropic or anisotropic materials, the influences of voids on the effective properties have been studied by many authors (Christensen, 1993; Jasiuk et al., 1994). The voided material, a typical heterogeneous material, consists of clearly distinguishable constituents or phases that show different physical material properties. The behaviour of inhomogeneous materials is determined by the relevant material properties of the constituents and by their geometry and topology. Inhomogeneous materials can be studied at a number of length scales ranging from the sub-atomic scales to the scales for which continuum descriptions are best suited.

An engineering analysis and design of porous solids requires a homogenization approach since numerical computations of macro-scale structures are extremely expensive if the full micro-structural topology is to be modelled. Via the homogenization, the response on the macro level includes microstructural features of the material behaviour. Many analytical approaches such as Mori-Tanaka model (Mori and Tanaka, 1973; Benveniste, 1987) are utilized to get effective material properties. These schemes can be applied to problems such as the determination of the effective thermal conductivity of porous rocks in partially saturated conditions (Gruescu et al., 2007). In the present paper, a pure numerical approach has been developed to evaluate effective material properties in voided heat conducting ceramic material. Numerical analyses are performed on the representative volume element (RVE) (Hill, 1963). The RVE contains sufficient microstructural information to be a representative of any similar volume taken from any location in the voided solid. 
In the present paper, the scaled boundary finite element method (SBFEM) is developed for 2D boundary value problem in a porous heat conducting solid under stationary boundary conditions. The eigenvalue decomposition is used to solve the scaled boundary finite element equation (Song and Wolf, 1997). The temperature field is expressed as a series of power functions of the radial coordinate. The SBFEM is applied to a numerical solution of the specific boundary value problems in the RVE of the material with voids to compute effective thermal conductivities. The present analyses give additional information concerning how the homogenized thermal conductivities of the material vary in terms of porosity and also in terms of pore shape.

\section{Governing equation of heat conduction}

The stationary heat transfer by conduction to calculate the temperature $T(\mathbf{x})$, at a point $\left(\mathbf{x}=(x, y)=\left(x_{1}, x_{2}\right)\right)$ of the spatial 2D solid domain is given by the generalized Laplace equation in Cartesian coordinates:

$$
k_{i j} T_{, i j}(x, y)=0
$$

where $k_{i j}$ is the thermal conductivity tensor. Equation (1) can be rewritten into the matrix form

$$
\{L\}^{T}\{q\}=0
$$

where the linear differential operator $\{L\}$ is given as

$$
\{L\}=\left\{\begin{array}{l}
\frac{\partial}{\partial x} \\
\frac{\partial}{\partial y}
\end{array}\right\}
$$

and the vector of heat flux can be obtained as

$$
\{q\}=[k]\{L\} T(x, y) \text {. }
$$

For orthotropic material one can define thermal conductivity matrix $[k]$ as

$$
[k]=\left[\begin{array}{cc}
-k_{11} & 0 \\
0 & -k_{22}
\end{array}\right]
$$

The following essential and natural boundary conditions are assumed on the global boundary $\Gamma$ :

$$
\begin{aligned}
& T(x, y)=\tilde{T}(x, y) \quad \text { on } \quad \Gamma_{T}, \\
& q(x, y)=-k_{i j} T_{, j}(x, y) n_{i}(x, y)=\tilde{q}(x, y) \quad \text { on } \quad \Gamma_{q},
\end{aligned}
$$

where $n_{i}(x, y)$ is the unit outward normal vector on the global boundary, $\Gamma_{T}$ is the part of the global boundary with the prescribed temperature and on $\Gamma_{q}$ the heat flux $\tilde{q}(x, y)$ is prescribed. 


\section{Scaled boundary finite element method for heat conduction}

In the present paper, the scaled boundary finite element method (SBFEM) is introduced to calculate the temperature field in heat conduction problems. The basic concept of SBFEM is shown in Figure 1. In this method, a scaling centre $O$ is selected at a point from which the whole boundary is directly visible. By scaling the boundary $S$ in the radial direction with respect to the scaling centre $O$ with a scaling factor $\xi$ which is smaller than 1 for bounded domain and larger than 1 for unbounded domain, the whole analyzed domain is covered.

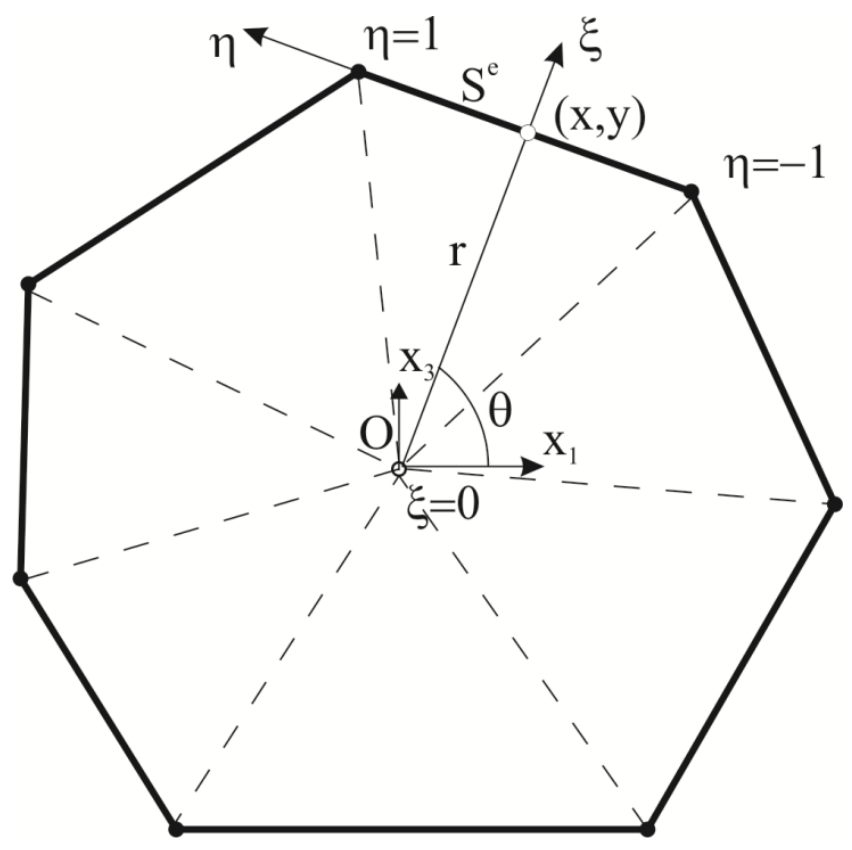

Figure 1 . The scaled boundary polygon representation

Figure 1 illustrates the basic idea of the scaled boundary finite element method for a 2D problem with domain $V$. Only the boundary $S$ of the domain is discretized with line elements $S^{e}$ when applying the scaled boundary finite element method. Then, the whole analyzed domain $V$ is decomposed into triangular sectors $V^{e}$ associated with the boundary line elements $S^{e}$. The global Cartesian coordinates $(x, y)$ of a point of a line element on the boundary $S^{e}$ (superscript $e$ represents element) are parameterized as

$$
x(\eta)=[N(\eta)]\{x\}, \quad y(\eta)=[N(\eta)]\{y\},
$$

with $\eta$ being the local (circumferential) coordinate $\eta \in[-1,1],[N(\eta)]=\left(N_{1}(\eta), N_{2}(\eta)\right)$ is the $1 \times 2$ matrix of shape functions $N_{1}(\eta)=(1-\eta) / 2, N_{2}(\eta)=(1+\eta) / 2$, and $\{x\},\{y\}$ are $2 \times 1$ vectors composed of the Cartesian coordinates of two nodal points on the boundary element, i.e. 


$$
\{x\}=\left(x^{1}, x^{2}\right)^{T}=(x(\eta=-1), x(\eta=1))^{T}, \quad\{y\}=\left(y^{1}, y^{2}\right)^{T}=(y(\eta=-1), y(\eta=1))^{T} .
$$

The analyzed domain is described by scaling the boundary with the dimensionless radial coordinate $\xi$ pointing from the scaling centre $O(\xi=0)$ to a point on the boundary $(\xi=1)$. The Cartesian coordinates $\left(x_{1}, x_{2}\right)$ of a point inside the domain are parameterized as

$$
\begin{aligned}
& x_{1}(\xi, \eta)=\xi x(\eta)=\xi[N(\eta)]\{x\} \\
& x_{2}(\xi, \eta)=\xi y(\eta)=\xi[N(\eta)]\{y\}
\end{aligned}
$$

where $\xi$ and $\eta$ are called the scaled boundary coordinates.

The scaled boundary coordinates in two dimensions resemble the polar coordinates. The polar coordinates in Figure 1 are expressed as

$$
\begin{aligned}
& r(\xi, \eta)=\xi r\{\eta\}=\xi \sqrt{x^{2}(\eta)+y^{2}(\eta)} \\
& \theta(\eta)=\arctan \frac{y(\eta)}{x(\eta)}
\end{aligned}
$$

The transformation between the components of the gradient operator in the Cartesian coordinate system and scaled boundary coordinate system is shown as

$$
\left\{\begin{array}{c}
\frac{\partial}{\partial \xi} \\
\frac{\partial}{\partial \eta}
\end{array}\right\}=[\hat{J}(\xi, \eta)]\left\{\begin{array}{c}
\frac{\partial}{\partial x_{1}} \\
\frac{\partial}{\partial x_{2}}
\end{array}\right\} \quad, \quad\left\{\begin{array}{c}
\frac{\partial}{\partial x_{1}} \\
\frac{\partial}{\partial x_{2}}
\end{array}\right\}=[\hat{J}(\xi, \eta)]^{-1}\left\{\begin{array}{c}
\frac{\partial}{\partial \xi} \\
\frac{\partial}{\partial \eta}
\end{array}\right\}
$$

with the Jacobian matrix defined as

$$
[\hat{J}(\xi, \eta)]=\left[\begin{array}{ll}
x_{1, \xi} & x_{2, \xi} \\
x_{1, \eta} & x_{2, \eta}
\end{array}\right]
$$

where $x_{1, \xi}, x_{2, \xi}, x_{1, \eta}$ and $x_{2, \eta}$ are determined from equations (6) and (7). Then, $[\hat{J}(\xi, \eta)]$ is rewritten into the factorized form

$$
\begin{aligned}
& {[\hat{J}(\xi, \eta)]=\left[\begin{array}{ll}
1 & 0 \\
0 & \xi
\end{array}\right]\left[\begin{array}{cc}
x(\eta) & y(\eta) \\
x(\eta)_{, \eta} & y(\eta)_{, \eta}
\end{array}\right]=\left[\begin{array}{ll}
1 & 0 \\
0 & \xi
\end{array}\right][J(\eta)],} \\
& {[\hat{J}(\xi, \eta)]^{-1}=[J(\eta)]^{-1}\left[\begin{array}{cc}
1 & 0 \\
0 & 1 / \xi
\end{array}\right]}
\end{aligned}
$$

with the radial coordinate $\xi$ being separated from the local coordinate $\eta$ on the boundary. The matrix $[J(\eta)]$ is the Jacobian matrix on the boundary $(\xi=1)$, i.e.

$$
[J(\eta)]=[\hat{J}(1, \eta)]=\left[\begin{array}{cc}
x(\eta) & y(\eta) \\
x(\eta)_{, \eta} & y(\eta)_{, \eta}
\end{array}\right],[J(\eta)]^{-1}=\frac{1}{|J(\eta)|}\left[\begin{array}{cc}
y(\eta)_{, \eta} & -y(\eta) \\
-x(\eta)_{, \eta} & x(\eta)
\end{array}\right]
$$

and its determinant is 
$|J(\eta)|=x(\eta) y(\eta)_{, \eta}-y(\eta) x(\eta)_{, \eta}$.

The linear differential operator $[L]$ in equation (3) is transformed to the coordinates $\xi, \eta$ as

$$
[L]=\left[b^{1}(\eta)\right] \frac{\partial}{\partial \xi}+\frac{1}{\xi}\left[b^{2}(\eta)\right] \frac{\partial}{\partial \eta}
$$

with

$$
\begin{aligned}
& {\left[b^{1}(\eta)\right]=\frac{1}{|J(\eta)|}\left[\begin{array}{c}
y(\eta)_{, \eta} \\
-x(\eta)_{, \eta}
\end{array}\right],} \\
& {\left[b^{2}(\eta)\right]=\frac{1}{|J(\eta)|}\left[\begin{array}{c}
-y(\eta) \\
x(\eta)
\end{array}\right] .}
\end{aligned}
$$

The temperature field $T(\xi, \eta)$ at any point inside the domain $V_{e}$ is obtained by interpolating $T(\xi)$ as with the shape functions $N^{e}(\eta)$ as follows

$$
\left.\{T(\xi, \eta)\}\right|_{V^{e}}=\left[N^{e}(\eta)\right]\{T(\xi)\} .
$$

Substituting equations (15) and (18) into equation (4) leads to

$$
\left.\{q(\xi, \eta)\}\right|_{V^{e}}=[k]\left[B^{1 e}(\eta)\right]\{T(\xi)\}_{, \xi}+\frac{1}{\xi}[k]\left[B^{2 e}(\eta)\right]\{T(\xi)\},
$$

where $\left[B^{1 e}(\eta)\right]=\left[b^{1}(\eta)\right]\left[N^{e}(\eta)\right],\left[B^{2 e}(\eta)\right]=\left[b^{2}(\eta)\right]\left[N_{, \eta}^{e}(\eta)\right]$.

The scaled boundary finite element equation for temperature field is derived as (Song and Wolf, 1997; Li et al., 2015)

$$
\xi^{2}\left[E^{0}\right]\{T(\xi)\}_{, \xi \xi}+\xi\left(\left[E^{0}\right]-\left[E^{1}\right]+\left[E^{1}\right]^{T}\right)\{T(\xi)\}_{, \xi}-\left[E^{2}\right]\{T(\xi)\}=0
$$

where the coefficient matrices $\left[E^{0}\right],\left[E^{1}\right],\left[E^{2}\right]$ are formed by assembling the element coefficient matrices

$$
\begin{aligned}
& {\left[E^{0}\right]=-\sum_{e=1}^{n} \int_{-1}^{+1}\left[B^{1 e}\right]^{T}[k]\left[B^{1 e}\right]|J(\eta)| d \eta} \\
& {\left[E^{1}\right]=-\sum_{e=1}^{n} \int_{-1}^{+1}\left[B^{2 e}\right]^{T}[k]\left[B^{1 e}\right]|J(\eta)| d \eta} \\
& {\left[E^{2}\right]=-\sum_{e=1}^{n} \int_{-1}^{+1}\left[B^{2 e}\right]^{T}[k]\left[B^{2 e}\right]|J(\eta)| d \eta}
\end{aligned}
$$

The heat flux through the boundary is equilibrated in the discretized form by the vector $\{R(\xi=1)\}$ corresponding to

$$
\{R(\xi)\}=\xi\left[E^{0}\right]\{T(\xi)\}_{, \xi}+\left[E^{1}\right]\{T(\xi)\}
$$

that is defined at interior points which are having the character of density of heat sources.

The solutions for $T(\xi)$ in Eq. (20) are expressed in a power function by following the eigenvalue decomposition technique (Song and Wolf, 1997) for the bounded domain as 


$$
\{T(\xi)\}=\sum_{i=1}^{n_{t}}\left\{\Psi_{i}^{(t)}\right\} \xi^{-\lambda_{i}^{t}} c_{i}
$$

where $n_{t}$ is the total number of nodes, $\lambda_{i}^{t}$ are the negative eigenvalues and $\left\{\Psi_{i}^{(t)}\right\}$ are the corresponding eigenvectors of standard eigenproblem. They can be interpreted as independent temperature modes. Substituting Eq. (23) into Eq. (19) one obtains the approximation of the heat flux vector within the sector $V_{e}$

$$
\left.\{q(\xi, \eta)\}\right|_{V_{e}}=\left.\sum_{i=1}^{n_{t}}\left\{\Psi_{q i}(\eta)\right\}\right|_{S_{e}} \xi^{-\lambda_{i}^{t}-1} c_{i}
$$

where the heat flux modes are defined as

$$
\left\{\Psi_{q i}(\eta)\right\}=\left.\sum_{e=1}^{n}\left\{\Psi_{q i}(\eta)\right\}\right|_{S_{e}},\left.\left\{\Psi_{q i}(\eta)\right\}\right|_{S_{e}}=[k]\left(-\left[B^{1 e}(\eta)\right]\left\{\Psi_{i}^{(t)}\right\} \lambda_{i}^{t}+\left[B^{2 e}(\eta)\right]\left\{\Psi_{i}^{(t)}\right\}\right)
$$

The integration constants $\{c\}$ are specified as follows

$$
\{c\}=\left[\begin{array}{lll}
\left\{\Psi_{1}^{(t)}\right\} & \cdots & \left\{\Psi_{N-1}^{(t)}\right\}
\end{array} \quad\left\{\Psi_{N}^{(t)}\right\}\right]^{-1}\{T(\xi=1)\}
$$

with $\{T(\xi=1)\}$ being the vector of nodal temperatures on the boundary (where $\xi=1$ ).

The conductivity matrix of individual subdomain is given according to (Song and Wolf, 1997) as

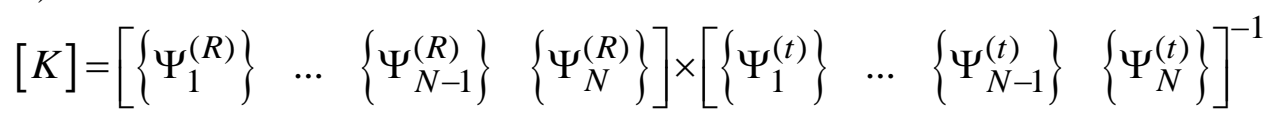

where $\left\{\Psi_{i}^{(R)}\right\}$ represents the heat source mode that is obtained from (22) by taking $\left\{\Psi_{1}^{(t)}\right\}$ instead of $\{T(\xi)\}$. The global conductivity matrix is assembled using the conductivity matrices of each subdomain that discretize the model. The standard finite element assemblage and solution procedures can be utilized at this point. The global system of equation is solved after enforcing appropriate boundary conditions in order to obtain the nodal temperature at the boundary $\{T(\xi=1)\}$ as

$$
\{T(\xi=1)\}=[K]^{-1}\{F\}
$$

with $\{F\}=\{R(\xi=1)\}$ being the vector given by prescribed heat fluxes at the boundary nodes.

\section{Computation of effective thermal conductivities}

Let us consider a rectangular RVE sample $\Omega=\left\{\forall \mathbf{x}=\left(x_{1}, x_{2}\right) ; x_{1}, x_{2} \in[0, w],\right\}$. Inside the square RVE domain there are generally distributed some micro-structural empty voids with arbitrary geometry. Then, the average values of the conjugated fields within the analysed sample are given as

$$
\left\langle q_{1}\right\rangle=\frac{1}{w^{2}}\left(\left.w \int_{0}^{w} q_{1}\right|_{x_{1}=w} d x_{2}+\int_{0}^{w}\left[\left.q_{2}\right|_{x_{2}=w}-\left.q_{2}\right|_{x_{2}=0}\right] x_{1} d x_{1}\right)
$$




$$
\left\langle q_{2}\right\rangle=\frac{1}{w^{2}}\left(\left.w \int_{0}^{w} q_{2}\right|_{x_{2}=w} d x_{1}+\int_{0}^{w}\left[\left.q_{1}\right|_{x_{1}=w}-\left.q_{1}\right|_{x_{1}=0}\right] x_{2} d x_{2}\right),
$$

and the integrands are obtained from the solution of considered boundary value problems.

If the boundary conditions are selected as shown in Figure 2, the average values of the secondary fields are given as

$$
\left\langle T_{, 1}\right\rangle=\vartheta_{1}=\text { const },\left\langle T_{, 2}\right\rangle=0 \text {. }
$$

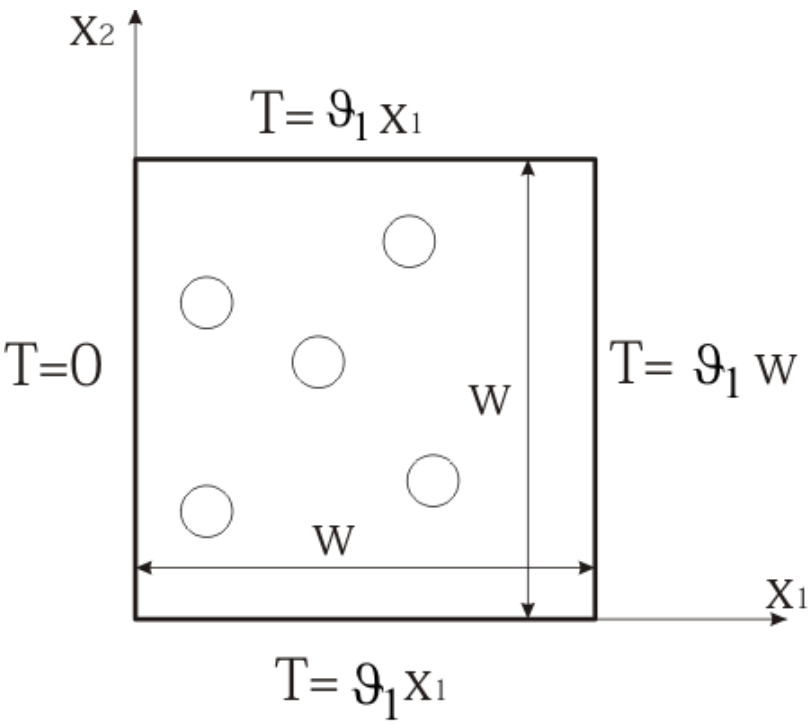

Figure 2. Boundary conditions appropriate for evaluation of $k_{11}^{\text {eff }}$

Then, we can get the following effective thermal conductivity

$$
k_{11}^{e f f}=-\frac{\left\langle q_{1}\right\rangle}{\vartheta_{1}}
$$

If the boundary conditions are selected as shown in Figure 3, the average values of the secondary fields are given as

$$
\left\langle T_{, 2}\right\rangle=\vartheta_{2}=\text { const },\left\langle T_{, 1}\right\rangle=0
$$




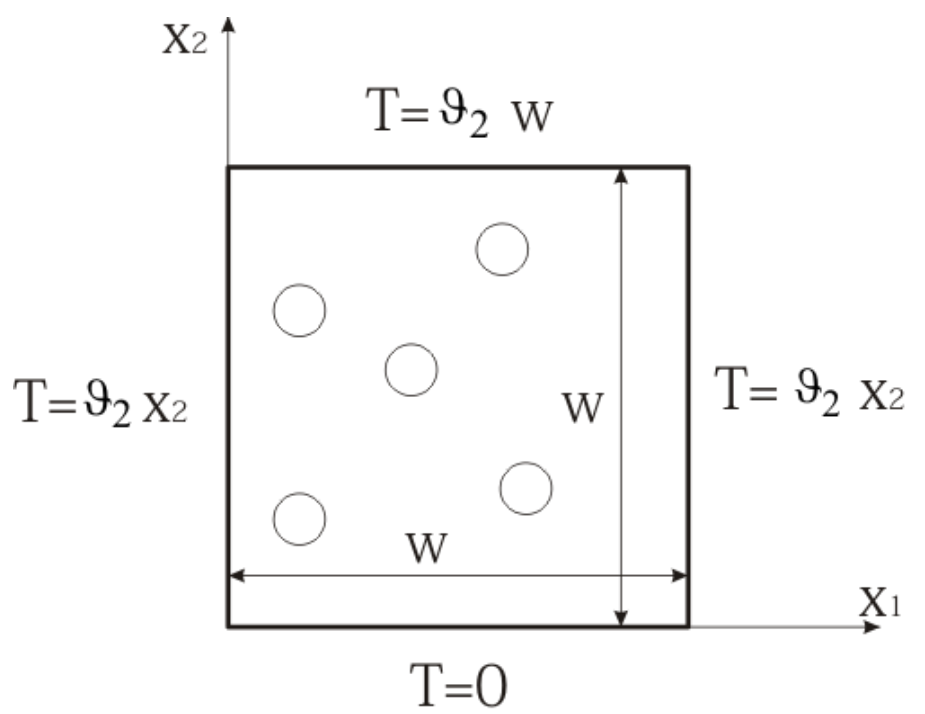

Figure 3. Boundary conditions appropriate for evaluation of $k_{22}^{e f f}$

Now, we can calculate the following effective material coefficients

$$
k_{22}^{e f f}=-\frac{\left\langle q_{2}\right\rangle}{\vartheta_{2}}
$$

where the average values of the conjugated fields $\left\langle q_{1}\right\rangle,\left\langle q_{2}\right\rangle$ are given by formulae (29), with the integrands being obtained from the solution of the considered boundary value problem.

Thus, having solved the above considered boundary value problems in the RVE sample; we can calculate all the effective thermal conductivity coefficients in materials with empty voids.

\section{Numerical examples}

As a numerical example single circular and elliptic void in square domain $(w \times w)$ is analyzed, where various values of void radii are considered thus specifying various volume fractions of pores. Two ceramic materials are considered in order to show the effect of varying volume fraction of pores on thermal conductivities. The material parameters [Li at al. (2015)] corresponding to cadmium selenide ceramic material are given as $k_{11}=50 \mathrm{WK}^{-1} \mathrm{~m}^{-1}, k_{22}=75$ $\mathrm{WK}^{-1} \mathrm{~m}^{-1}$ and for alumina $\left(\mathrm{Al}_{2} \mathrm{O}_{3}\right) k_{11}=35 \mathrm{WK}^{-1} \mathrm{~m}^{-1}, k_{22}=39 \mathrm{WK}^{-1} \mathrm{~m}^{-1}$.

Since the RVE is not a simply-connected domain, it is divided into polygons and each polygon is treated as a scaled boundary finite element subdomain (see Fig. 1). There are needed at least 4 subdomains to solve this problem. We have considered 12 polygons in numerical analyses (Fig. 4) with getting quasi uniform fictitious triangulation which is appropriate for approximation accuracy. Only boundaries of subdomains need to be discretized with line elements. Three nodes elements are used for discretization of subdomain boundaries. There are totally 108 degrees of freedom (DOFs) in this mesh. 

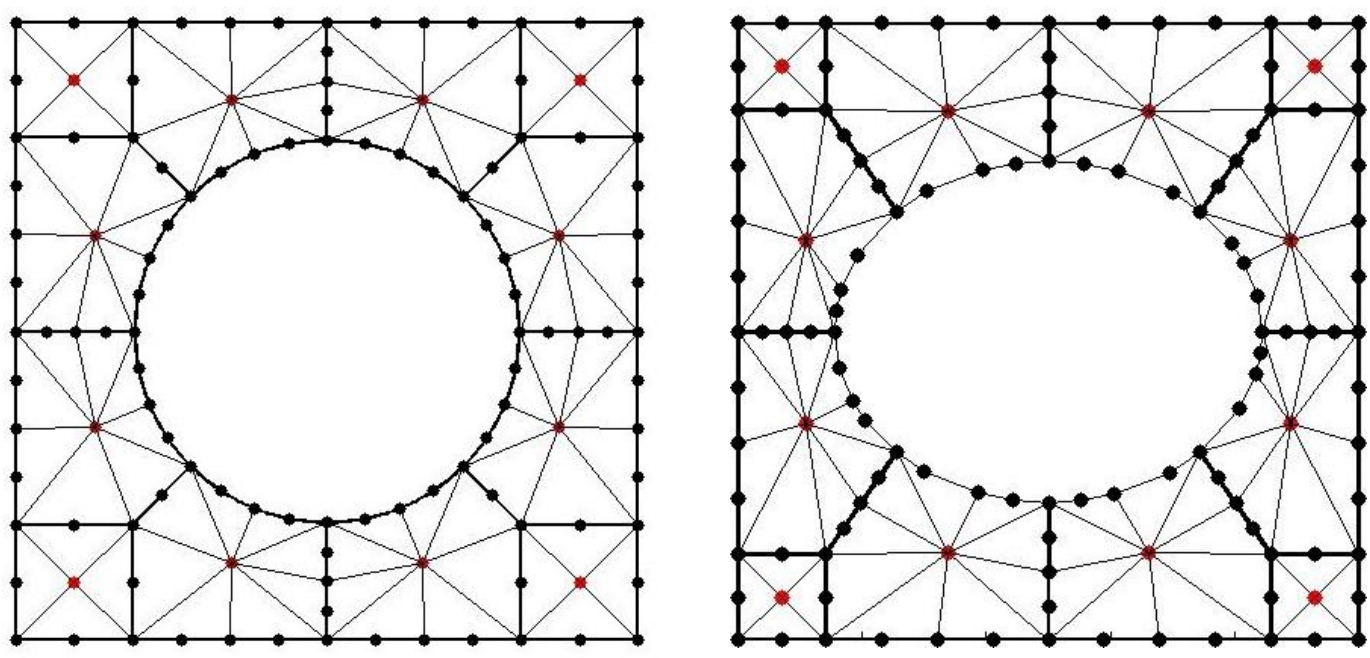

Figure 4. Discretization of a square domain with a circular and elliptic void in the SBFEM

For comparative purposes we have analyzed the same problem by the finite element method (FEM). The software COMSOL has been applied. We used very fine mesh, where various numbers of elements have been considered in dependence on the radius of the circular void. Total number of elements 9689 and 5240 has been used for minimum porosity (volume fraction of 0.05 ) and maximum porosity with 0.5 , respectively. The volume fraction of voids for the circular void is defined as $f=\pi r_{0}^{2} / w^{2}$, where $r_{0}$ is the radius of the circular void. For the elliptic void one gets $f=\pi a b / w^{2}$, where $a$ and $b$ are its major and minor semi-axes. Aspect ratio characterizing the shape of an ellipse is given as $c=b / a$.

The numerical results for effective material parameters are presented in Figures $5-8$. The fixed numbers of discretized lines by nodes were used in SBFEM for various radii of circular and elliptic voids. Three aspect ratios are considered assuming $c=1.0$ represents a circular void. Note that due to the flattening of an ellipse with $c=0.5$, volume fraction of voids is limited to $f=0.3$. The effective thermal conductivities $k_{11}^{\text {eff }}$ and $k_{22}^{\text {eff }}$ are shown in Figure 5 and 6, respectively for cadmium selenide and in Figure 7 and 8 for alumina, respectively. They have a similar decreasing tendency with growing porosity. One can observe that the shape of void has a significant effect on effective thermal conductivities. While the values of $k_{11}^{e f f}$ decrease less compared to the circular void, the values of $k_{22}^{\text {eff }}$ show significant decrease for smaller aspect ratio. Very good agreement between SBFEM and FEM results is observed. 


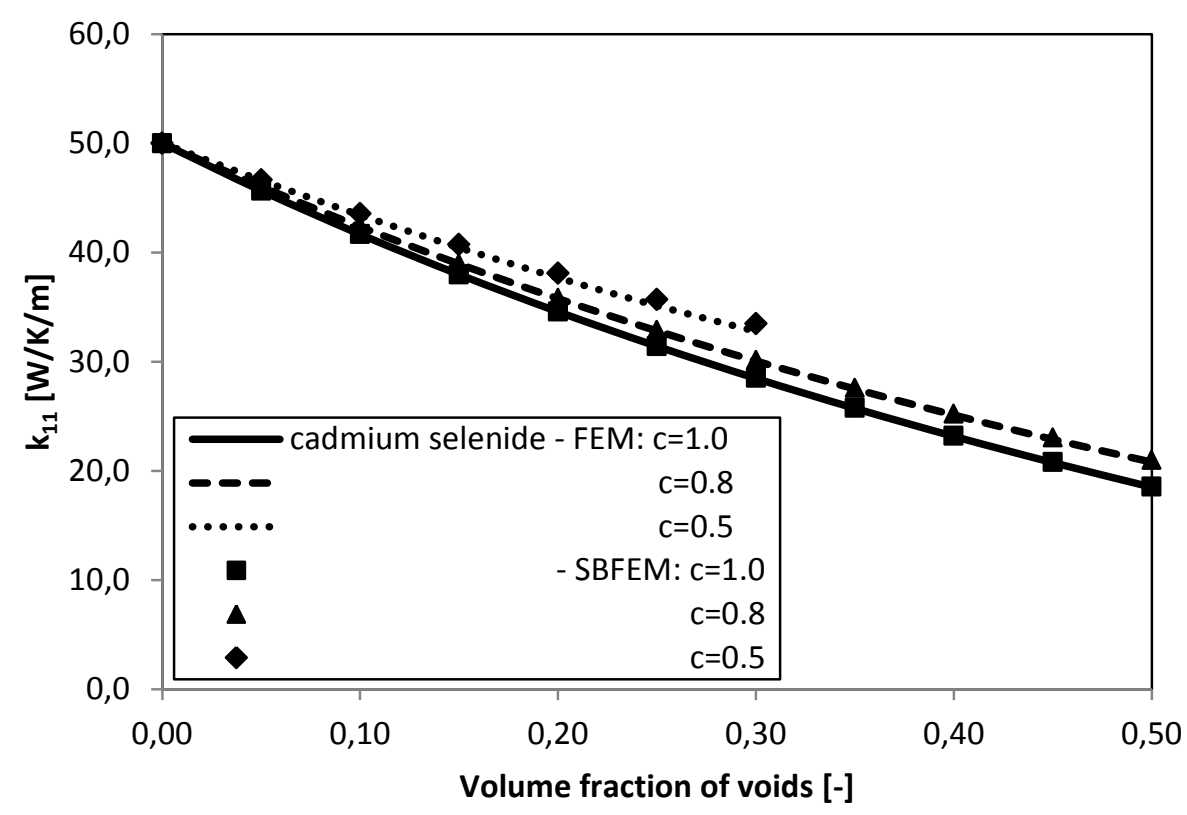

Figure 5. Variation of effective thermal conductivities $k_{11}^{\text {eff }}$ on porosity for cadmium selenide

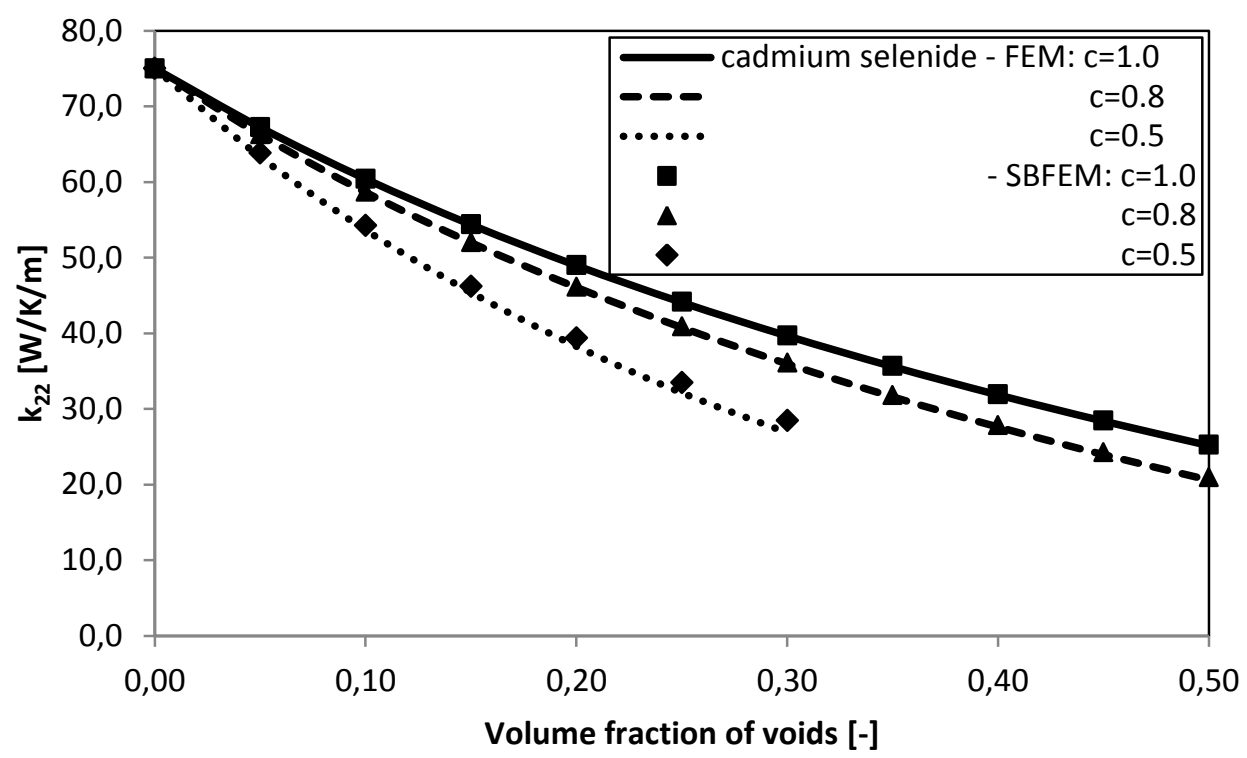

Figure 6. Variation of effective thermal conductivities $k_{22}^{\text {eff }}$ on porosity for cadmium selenide 


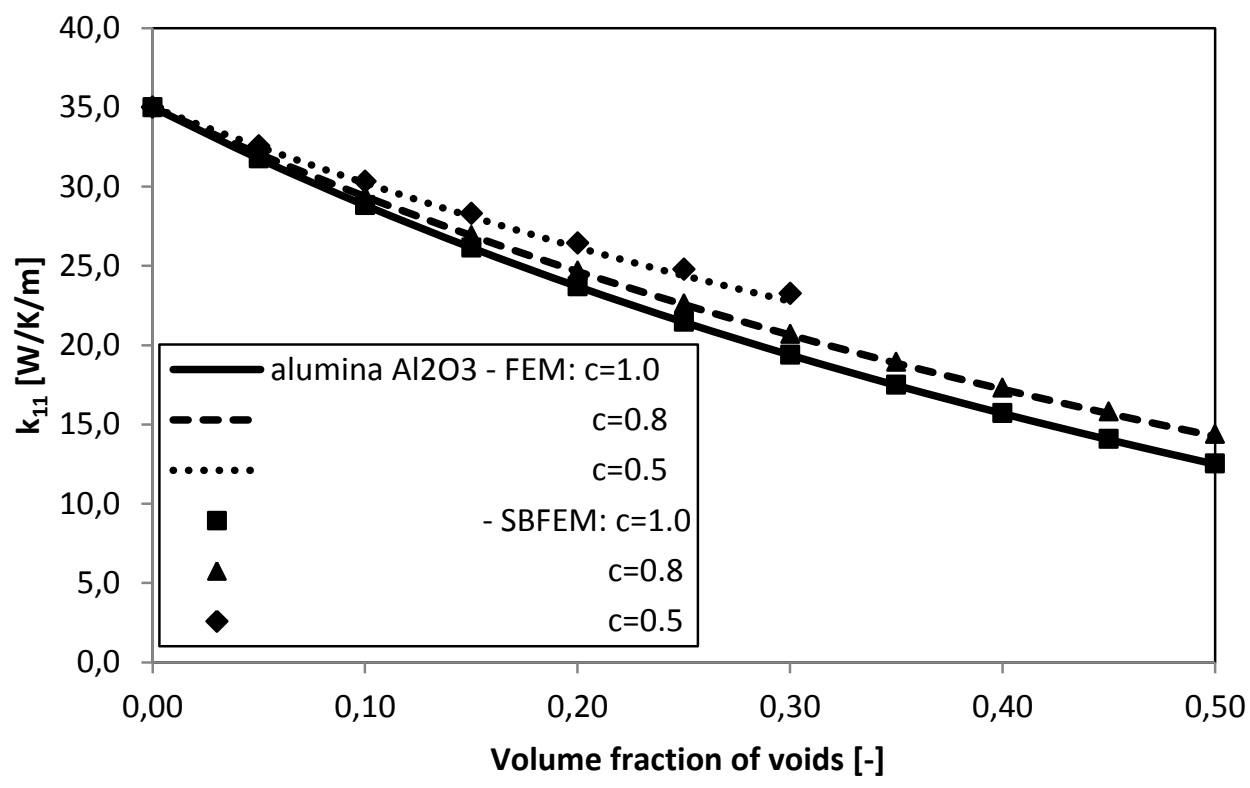

Figure 7. Variation of effective thermal conductivities $k_{11}^{e f f}$ on porosity for alumina

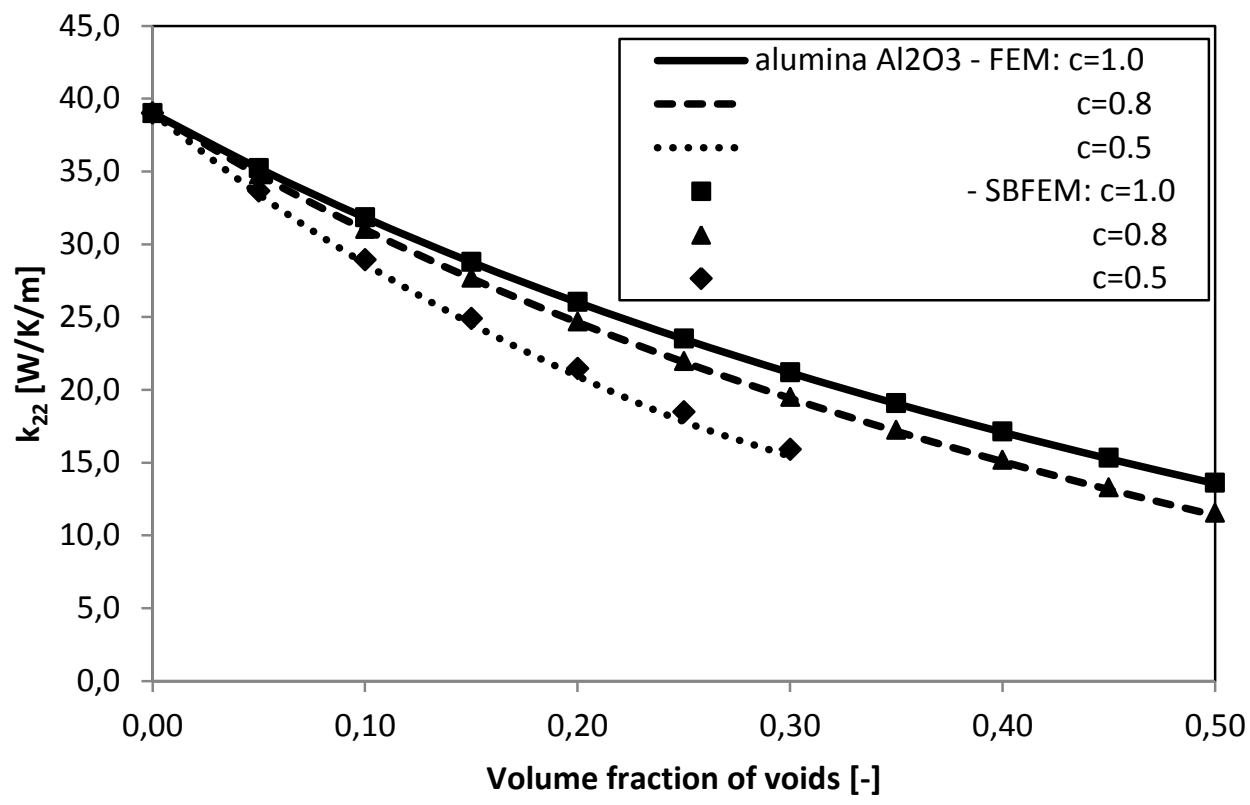

Figure 8. Variation of effective thermal conductivities $k_{22}^{\text {eff }}$ on porosity for alumina 


\section{Conclusions}

The scaled boundary finite element method has been applied to solve heat conduction problems for the RVE to get effective material properties of the material with voids. For the reliable functioning and design of voided materials it is necessary to use advanced numerical methods. The applicability of the proposed numerical method is demonstrated by the numerical examples assuming square RVE with a central circular pore. Obtained effective thermal conductivities decrease significantly with a growing volume fraction of pores. For elliptic pore different trends are observed for thermal conductivities depending on the aspect ratio of an elliptic void.

The numerical results are compared with the finite element method results and a very good agreement is observed. Applicability, accuracy and efficiency of the SBFEM method are clearly demonstrated.

\section{Acknowledgement}

The authors acknowledge the support by the Slovak Science and Technology Assistance Agency registered under the number APVV-0014-10 and the Slovak Grant Agency VEGA2/0011/13.

\section{References:}

[1] Abbasbandy, S. and Shirzadi, A. (2010), "A meshless method for two-dimensional diffusion equation with an integral condition”, Eng Anal Bound Elem 34, 1031-1037.

[2] Abreu, A. I., Canelas, A. and Mansur, W. J. (2013), “A CQM-based BEM for transient heat conduction problems in homogenous materials and FGMs", Applied Mathematical Modelling 37, 776-792.

[3] Atluri, S. N. (2004), The meshless method (MLPG) for domain and BIE discretizations, Tech Science Press, Forsyth, USA.

[4] Bathe, K. J. (1976), Numerical Methods in Finite Element Analysis, Prentice-Hall, New Jersey, USA.

[5] Benveniste, Y. (1987), "A new approach to the application of Mori-Tanaka's theory in composite materials", Mechanics of Materials 6 (2), 147-157.

[6] Brebbia, C. A., Telles, J. C. and Wrobel, L. C. (1984), Boundary Elements Techniques: Theory and Applications in Engineering, Springer-Verlag, Berlin-New York.

[7] Cai, Z., Mandel, J. and McCormick, S. (1991), "The finite volume element method for diffusion equations on general triangulations", SIAM Journal on Numerical Analysis 28, 392-403.

[8] Carslaw, HS. and Jaeger, J. C. (1959), Conduction of Heat in Solids, Oxford University Press, Vivian Ridler, Walton Street, Oxford, second edition.

[9] Gruescu, C., Giraud, A., Homand, F., Kondo, D. and Do, D. P. (2007), "Effective thermal conductivity of partially saturated porous rocks", International Journal of Solids and Structures 44, 811-818. 
[10] Hill, R. (1963), "Elastic properties of reinforced solids: some theoretical principles", Journal of the Mechanics and Physics of Solids 11 (5), 357-372.

[11] Christensen, R. M. (1993), "Effective properties of composite materials containing voids". Proceedings of the Royal Society A, 440 (1909), 461-473.

[12] Jasiuk, I., Chen, C. and Thorp, M. F. (1994), "Elastic moduli of two dimensional materials with polygonal and elliptical holes", Applied Mechanics Review 47, 1S, $18-28$.

[13] Juncu, Gh. (2008), "Unsteady conjugate forced convection heat/mass transfer from a finite flat plate", International Journal of Thermal Sciences 47, 972-984.

[14] Li, C., Ooi, E.T, Song, C. and Natarajan, S. (2015), "SBFEM for fracture analysis of piezoelectric composites under thermal load", Int J Solids Struct, 52, 114-129.

[15] Mori, T. and Tanaka, K. (1973), "Average Stress in the Matrix and Average Elastic Energy of Materials with Misfitting Inclusions", Acta metallica 21, 571-574.

[16] Ochiai, Y. (2001), "Steady heat conduction analysis in orthotropic bodies by triplereciprocity BEM", Computer Modeling in Engineering and Sciences 2(4), 435-446.

[17] Ozisik, M. N. (1994), Finite Difference Methods in Heat Transfer, CRC Press Inc, Boca Raton, USA.

[18] Sladek, J., Sladek, V., Solek, P., Tan, C. L. and Zhang, Ch. (2009), "Two- and three-dimensional transient thermoelastic analysis by the MLPG method", CMES: Computer Modeling in Engineering \& Sciences 47, 61-95.

[19] Sladek, J., Sladek, V., Zhang, Ch. and Tan, C. L. (2006), "Meshless local PetrovGalerkin method for linear coupled thermoelastic analysis", CMES: Computer Modeling in Engineering \& Sciences 16, 57-68.

[20] Sladek, J., Sladek, V., and Zhang, Ch. (2003), "Transient heat conduction analysis in functionally graded materials by the meshless local boundary integral equation method", Comput Mat Sci 28, 494-504.

[21] Sladek, J., Stanak, P., Han, Z. D., Sladek, V. and Atluri, S. N. (2013), "Applications of the MLPG Method in Engineering \& Sciences: A Review", CMES: Computer Modeling in Engineering \& Sciences 92(5), 423-475.

[22] Song, C. and Wolf, J. (1997), "The scaled boundary finite-element method- alias consistent infinitesimal finite-element cell method- for elastodynamics", Comput Methods Appl Mech Engng 147, 329-355.

[23] Song, C., (2006), "Analysis of singular stress fields at multi-material corners under thermal loading", Int J Numer Methods Eng 65(5), 620-652. 